\title{
Infectious spleen and kidney necrosis virus (ISKNV) used a novel strategy to modulate the mitochondria/ caspases -mediated cell death pathway via ROS signaling in a fish cell line
}

Pin-Han Chen ${ }^{1}$, Tsai-Ching Hsueh ${ }^{1}$ and Jiann-Ruey Hong ${ }^{1,2 \S}$

${ }^{1}$ Laboratory of Molecular Virology and Biotechnology, Institute of Biotechnology, National Cheng Kung
University, Tainan, Taiwan.
${ }^{2}$ Department of Biotechnology and Bioindustry Sciences, National Cheng Kung University, Tainan, Taiwan.

\begin{abstract}
Iridoviruses are large DNA viruses that infect invertebrates and poikilothermic vertebrates, such as insects, fish, amphibians, reptiles, crustaceans, and mollusks. The emergence of deadly iridoviral disease in aquatic fish has led to a growing interest in the mechanism of iridovirus-induced host cell death. In this present, we found that infectious spleen and kidney necrosis virus (ISKNV) can trigger a host cellular death via ROS signaling in grouper fin (GF-1) cells. ISKNV infection can induce reactive oxygen species (ROS) production by using $\mathrm{H}_{2}$ DCFDA assay at early replication stage. Then, this ROS signaling can upregulate the pro-apoptotic genes Bax and Bak expressions at middle replication stage. Moreover, ISKNV-induced ROS-mediated signals triggered the mitochondrial membrane potential (MMP) loss, which correlated to activate downstream caspases such as caspase-8, caspase-9 and caspase-3 at late replication stage. Final, we found that antioxidants treatment dramatically reduced ISKNV-induced host cellular death in GF-1 cells. Taken together our data show that ISKNV used a novel strategy to regulate the mitochondria/caspase-mediated cell death pathway via ROS signaling, which may provide new insight into DNA virus control and treatment.
\end{abstract}

Keywords: ISKNV, ROS signaling, Bax/Bak, Upregulation, Antioxidant

§Corresponding author. Tel.: +886-6-2003082; Fax: +886-6-2766505.

E-mail address: jrhong@mail.ncku.edu.tw. 